\title{
The Resection Map, a proposal for intraoperative hepatectomy guidance
}

\author{
P. Lamata - A. Jalote-Parmar - F. Lamata • \\ J. Declerck
}

Received: 10 January 2008 / Accepted: 17 May 2008 / Published online: 10 June 2008

(C) CARS 2008

\begin{abstract}
Objective To propose a new concept of an intra-operative $3 \mathrm{D}$ visualisation system to support hepatectomies. This system aims at improving the transfer of pre-operative planning into the intra-operative stage, both in laparoscopic and open approaches.

Materials and methods User (surgeon) centred developmental process to identify the surgical requirements is applied. The surgical workflow of hepatectomy is analyzed, including observations of liver surgeries and focus group sessions. Based on this analysis, specifications for the "Resection Map" are defined. A first implementation is developed, and preliminary clinical acceptance results are gathered.

Results Control of main veins and tumour margins are the two critical aspects. The "Resection Map" provides an intuitive visualisation of structures nearby the resection plane without any registration to the patient space. The first
\end{abstract}

P. Lamata $(\varangle) \cdot$ J. Declerck

Siemens Molecular Imaging,

23-38 Hythe Bridge Street, Oxford OX1 2EP, UK

e-mail: Pablo.Lamata@siemens.com

URL: http://www.medical.siemens.com, http://www.ariser.info

J. Declerck

e-mail: Jerome.Declerck@siemens.com

\section{A. Jalote-Parmar}

Industrial Design Department and Engineering,

Delft University of Technology,

Delft, The Netherlands

e-mail: a.jaloteparmar@tudelft.nl

URL: http://www.io.tudelft.nl/medisign

\section{F. Lamata}

Hospital Clínico Universitario of Zaragoza,

Avda. San Juan Bosco, 15, 50009 Zaragoza, Spain

e-mail: flamata@salud.aragon.es

URL: http://www.hcu-lblesa.es prototype subjectively increases the surgeon's confidence and orientation, but it should be further developed for non anatomical resections.

Conclusions The Resection Map is proposed as a pragmatic solution to enhance liver resection accuracy and safety.

Keywords Computer-assisted surgery · Hepatectomy · Equipment design

\section{Introduction}

Minimally invasive and robotic surgeries are constantly giving rise to new research and development. By extending the surgeon's ability to plan and carry out interventions more accurately and less invasively, computer aided surgical systems will address a crucial need to reduce medical costs, improve clinical outcomes and improve the efficiency of health care delivery. In this paper we focus on systems that are responsible for organisation, image processing and display of the complex interior structures of the liver which is to be used in resection procedures (hepatectomies). These systems allow the surgeon to orient him/herself by means of computer-generated visualisations of the anatomy, target structures and planned paths.

Major research work in computer aided surgical systems for liver surgery is focused on providing preoperative planning support [1-5]. These systems perform image segmentation and 3D reconstruction of liver, tumour, and vessel structures. They offer the edition and calculation of anatomical and non anatomical resections. Their resection proposals consider tumour size and position, the relation of the tumour to the vessel structure and user-defined security margins. User interaction is generally in 2D rendered images, or even involving a 3D virtual environment [3]. These systems 
are yet not focusing on the presentation of information intraoperatively, and the surgeon is forced to rely on his memory and ability to translate the preoperative figures into the operative site $[1,6,7]$.

Intraoperative guidance is currently provided with ultrasound (US). Laparoscopic US provides a very restricted field of view and only 2D images to date, and its utility decreases as resection progresses. In open surgery, the use of a multiplanar visualisation of 3D US with tracked tools has reported an improved accuracy in resections [8]. Advanced imaging modalities like the C-Arm [9] produce radiation, and are bulky and still available only in specialised operating rooms (ORs). Finally, efforts for registration of preoperative images relying on external fiducial markers [10] lack in clinical accuracy for intra-operative liver resection guidance. Due to lack of effective intraoperative visualisation systems, there exists an increased risk of trespassing safety tumour margins and cutting critical hidden structures, leading to potential harm to the patient and significant lengthening of the surgical procedure. Thus, there exists a need to enhance the safety of the surgical procedure by providing the surgeon with the localisation of the critical structures during liver resection.

The approach taken to address this clinical need is to present a Resection Map to the surgeon, a system for simplified and effective visualisation of the critical structures and the path that has been planned for the resection. The concept of providing a map in real-time tasks is similar to the use of a navigation system while driving a car, or to the use of context maps in computer games [11]. The focus here is in the optimal presentation and use of preoperative information during the surgical procedure.

The rest of the paper is organised as follows. Requirements for this Resection Map were reviewed in several surgical centres in the Netherlands, Norway and Spain by conducting user observations and focus groups with surgeons, the result of which is explained in detail in "Surgical requirements analysis". Based on this analysis and current possibilities of technology, specifications of the Resection Map are defined in "Design of the hepatic Resection Map". A first implementation developed using open source visualisation libraries, and some preliminary acceptance results are described in "Implementation and concept validation" section. "Discussion" is centered on the value of the proposed design and the need of positioning information of the surgical tools. Finally, some concluding remarks are outlined.

\section{Surgical requirements analysis}

To investigate the surgical requirements, an analyse of the surgical workflow was conducted by applying workflow analysis framework as proposed in [7]. The surgical workflow analysis included the following stages: (1) observation of liver surgeries $(n=20)$ including both open and endoscopic liver resection. The surgeons were observed in all the three phases of the surgeries (pre, intra and post); (2) One focus group session on endoscopic liver surgeries $(n=7)$. The focus group session involved investigating the current problems faced by the surgeons due to lack of proper visualisation in liver surgery. These studies were conducted in three national hospitals: Rikshospitalet (Oslo, Norway), Erasmus MC (Rotterdam, Netherlands) and Hospital Clínico (Zaragoza, Spain). The key findings related to our area of research are summarized in the following sections.

\section{Difficulties in liver resection}

A safe liver resection has to prevent uncontrolled bleeding of vessels. Veins, having weak walls and constant flow, are the vessels which lead to most dramatic surgical errors. On the contrary, arteries, with stronger walls, smaller diameter and pulsate flow, are more robust and therefore difficult to harm, and easier to locate and seal if perforated. The second important safety factor is to guaranty a minimum tissue margin around the tumour, when present.

Localisation of these inner structures is very hard to anticipate for a surgeon, who therefore resects the liver very carefully, step by step, looking for the next inner structure to appear. Moreover, identification of these hidden structures is difficult since the operating field becomes really confusing due to the presence of bleeding and burnt tissue. An illustration of the problem is provided in Fig. 1, which shows an internal view of a liver surgery.

Of second clinical importance is the preservation of the portal triad (portal veins, hepatic arteries and biliary ducts) and the hepatic veins during the surgical act. Damage of these structures causes a loss in the remaining functional liver. This

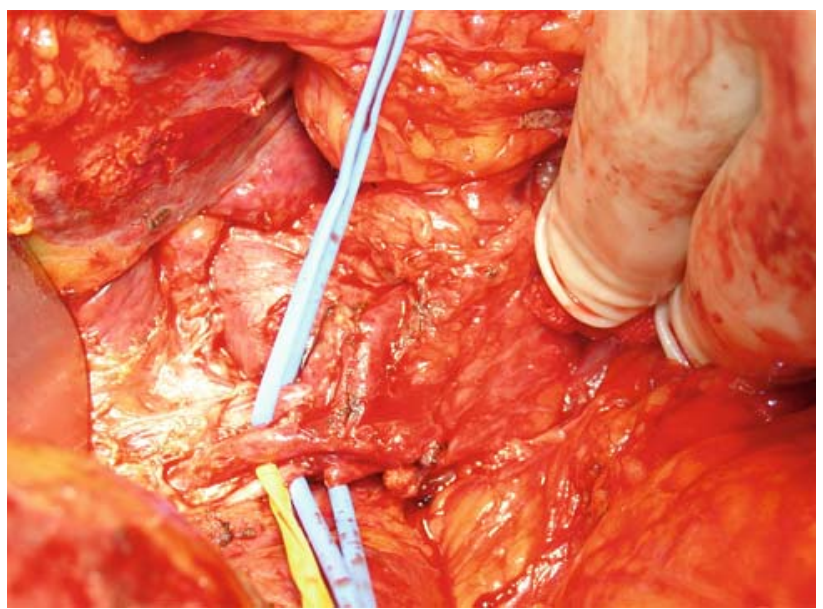

Fig. 1 Identification in open surgery of main vessels (portal vein, biliar duct and hepatic arteries) with strings. This is done preliminary to the resection, and could be regarded as the clearest picture that the surgeon will have of the liver vessels 
may not always be critical since liver is an organ with a great autoregeneration capability [12], and it mainly depends on the decisions taken in the planning stage.

Control of vessels is partially addressed with the use of specialized tools that resect the liver parenchyma respecting the inner vessels. Some examples are the Ultrasonic dissector (e.g. CUSA ${ }^{\circledR}$, Valleylab, Boulder, USA), harmonic scalpel (e.g. Ultracision ${ }^{\circledR}$, Johnson \& Johnson, USA) or the irrigated monopolar scalpel (e.g. Tissuelink ${ }^{\circledR}$, TissueLink Medical, Inc., USA). Once conducts are dissected, they are joined safely with vessels sealers (e.g. Ligasure Atlas ${ }^{\circledR}$, Valleylab, Boulder, USA) or vessel mechanical sutures (e.g. EndoGia ${ }^{\circledR}$, Tyco Healthcare, USA). But there is still a need to further enhance the safety and accuracy of the procedure by providing the surgeon with the localisation of the critical structures.

Abdominal surgery is even more challenging for a surgeon when the laparoscopic approach is taken. The surgeon has to adapt to the limited workspace, and to understand the anatomy seen from a laparoscope, with organs at different scales and orientations and distorted views. He/she needs to mentally match some specific anatomical information from preoperative imaging studies to the laparoscopic operating field. And the surgeon cannot palpate the liver stiffness to confirm the tumour location, as done in open surgery.

\section{Planning data limited intraoperatively}

Surgeons plan several resection scenarios before conducting the liver surgery, which includes main steps such as: planning resection strategy; identifying the critical structures to be avoided; and conducting volumetric analysis. Currently, planning information is not optimally transferred to the intraoperative stage in terms of visualising critical areas and navigation paths. Due to lack of visualising aids which can be transferred to the surgical theatre, the surgeons have to rely heavily on their memory. To our knowledge, there is no solution which focuses on providing an effective transfer of planning knowledge into the intra-operative phase. Some authors report the use of the preoperative tools in the intraoperative stage [13].

Need for 3D visualisation of risk information

An intraoperative visualisation system is especially required in two situations: (1) non anatomical resections, when resections do not follow the divisions between liver lobes, and (2) cases with anatomical variations in topological configuration of vessels. Laparoscopic liver resections are still very limited to tumours localized close to the surface, mainly dorsal and left lateral, and an effective navigation system could increase the number of liver tumours that can be treated minimalinvasively.
Specifically, such a system should provide the 3D visualisation of the resection plane and associated risk information: location of main veins and tumours. While cutting the parenchyma, surgeons could prevent errors and severe bleedings with a system that informs him/her when coming close to a vessel. 3D Visualisation of risk information in terms of distances between vessels and tumours in the path of resection could assist the surgeon in the navigation task.

Integrating systems into the existing surgical workflow

New guidance and visualisation tools often change current surgical workflow. Surgeons indicated that technological solutions that demand a large change in the existing workflow are difficult to adapt in surgical theatres. Such solutions are imposed rather than required by the surgeons. This implies the need to optimally merge the new computer aided systems with the existing surgical workflow.

\section{Design of the hepatic Resection Map}

Proposed concept of the intra-operative visualizing system

In abdominal surgery it is difficult to locate well defined anatomical landmarks or a reliable bone structure, as can be used in neurosurgery. Surgical dissection, ligament cuts, organ displacements, etc. continually transform the operating field. Automatic tracking of surgical targets and relative positioning of tools are thus a difficult challenge that prevents the registration of preoperative information to the patient space.

Therefore, the proposed solution is to offer an intraoperative $3 \mathrm{D}$ visualisation of the key internal liver structures without any registration to the patient space and, correspondingly, without the real-time positioning of surgical tools in the 3D visualisation environment. The "Resection Map" is a representation of the planned resection path through the organ showing the relevant structures and risk areas (see Fig. 2). This can be understood as a "surgical GPS map" that shows the route and its key landmarks to the physician without the information of knowing where the surgeon is in this map.

The surgeon can then visualize the hidden relevant structures (main vessels and tumours) that surround the resection path. The hypothesis is that this information enables the surgeon (1) to track his resection progress and (2) to identify the risk areas. The resection progress is inferred from the sequential identification of the key landmarks displayed in the Map, as what a pirate would do to find his buried treasure. This information on relative progress allows the surgeon to approximately mentally locate the tips of the tools in the Map, and to identify the proximity of a risk. 
Fig. 2 Basic concept design of the Resection Map to transfer the preoperative planning information to the intraoperative stage: a visualisation of relevant structures is used by the surgeon during the procedure as a Map that illustrates the path with the key landmarks he is going to follow. No registration to the surgical site is needed for it

\section{Preoperative Planning}

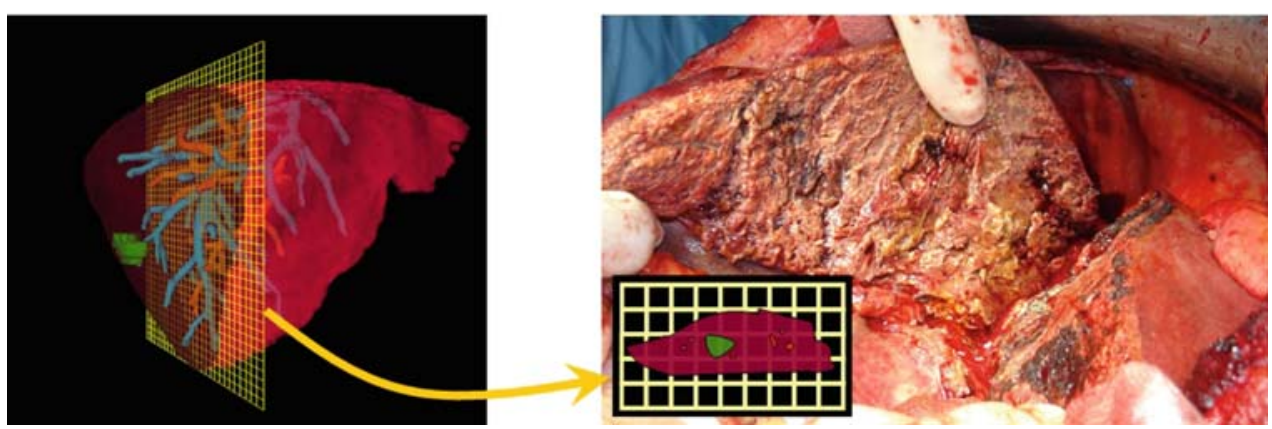

One kind of risk is the tumour proximity. Areas of the resection path which are closer to the tumour would be resected with caution of not diverting towards the direction of the tumour in order to prevent undesired tumour cell contact. The second important risk during resection is the damage of an important vein. This is especially likely to occur when veins are tangential to the plane of the resection, when a small mistake can cause a big longitudinal cut in the vein wall. The relative orientation of proximal veins with respect to the resection plane is thus an important piece of risk information that is provided in the Resection Map.

One third safety factor is the respect of other vascular structures: arteries and bile ducts. Damage to their integrity can reduce subsequent liver function, but this mainly depends on preoperative planning decisions. Intraoperative risks associated to these structures are unlikely to occur. On the other hand, these vascular structures are mainly located along the portal triad and thus they do not provide more landmarks to follow progress. Moreover, technically it is much more difficult to offer a clean visualisation of these small structures. Therefore arteries and bile ducts are not included in the Resection Map, but could be considered for forthcoming improvements.

\section{Information visualisation and components}

A proposed design for the Resection Map is described in Tables 1 and 2 in terms of its structural and visualisation components. A detailed description is provided in following paragraphs.

The first structural component is the Resection Plane. It is the 3D surface of the optimal path defined by the surgeon to perform the resection. It can be flat or curved, depending on the planning decision of the surgeon in the preoperative stage. This plane shows a $2 D$ grid of $1 \mathrm{~cm}$ to provide some distance references.

Only main veins represent a significant risk of uncontrolled bleeding during the surgery. And only big veins can be visually identified by surgeons during the progress of the resection. Based on the surgical observations, the minimum diameter defined for visualisation of veins is set to $3 \mathrm{~mm}$.
Only the vein segments near the Resection Plane are represented with its 3D structure. The definition of this Resection Window for visualisation was agreed with surgeons to be a slice of $3 \mathrm{~cm}$ surrounding the Resection Plane $(1.5 \mathrm{~cm}$ on each side), considering the maximal potential deviation from the original path. This width could also be adjusted during the procedure. For a clearer visualisation, remaining veins are coloured in a darker shade than those that are going to be resected.

The key landmarks in the Resection Map are the points where main veins are intersected with the Resection Plane. These Vein Cutting Areas are then highlighted, and its topology helps to better identify the risk of tangential cuts in veins. Another important reference is the contour of the liver; the Liver Cutting Area is therefore also highlighted. Tumours, critical structures to control, are represented with their 3D structure and always visible. Finally, the Resection Map also illustrates the resection entry point and the direction that the surgeon defined, what defines the Resection Route.

The visualisation should be clear and intuitive, allowing immediate mapping of the actual surgical view with the Resection Map. It should also clearly show the relevant localisation information. Our proposed solution combines two views, each of them focused in each of these two aspects.

The first, the Surgical View, contains the same point of view that the surgeon is expected to have during the procedure. It represents all the contents of the Map in opaque colours. A Progress Window is introduced to select structures at a certain depth, at a certain resection region, and to provide a clear visualisation of them. Otherwise, vascular structures would occlude each others. This 3D window is a box that defines in this Surgical Viewwhich structures are faded (behind the box), and which are removed (ahead the box). It can slide following the Resection Route. This is an adaptation of the magic lenses concept used in [14].

The second, the Progress View, is a flat visualisation of the Resection Plane with the Resection Route, seen from a perpendicular direction. The contours of the structures cut by this plane, the liver and veins, are highlighted. It also includes the tumour and the 3D model of veins, although they could be disabled. It is thus the view to track the resection 
Table 1 Structural components of the proposed design for the Resection Map
Table 2 Visualisation components of the proposed design for the Resection Map

\begin{tabular}{|c|c|c|}
\hline Component & Information visualisation and function & Interaction \\
\hline Resection plane & 3D surface that defines the resection path through the liver & None \\
\hline Tumours & 3D geometry of tumours in green colour & None \\
\hline Veins & $\begin{array}{l}\text { 3D geometry of veins. Portal system is coloured in orange and } \\
\text { hepatic in blue. Veins sections to be resected are brighter, and } \\
\text { remaining ones are darker }\end{array}$ & Enable/disable \\
\hline Liver & $\begin{array}{l}\text { 3D geometry of the liver. Coloured in dark red with a high trans- } \\
\text { parency }\end{array}$ & None \\
\hline 2D grid & $\begin{array}{l}2 \mathrm{D} \text { squared grid of } 1 \mathrm{~cm} \text { that is mapped on the Resection Plane. It } \\
\text { provides information on the distances between structures }\end{array}$ & Enable/disable \\
\hline Vein cutting areas & $\begin{array}{l}\text { Intersection between the resection plane and veins, dilated to have } \\
\text { a thickness of } 0.3 \mathrm{~cm} \text {. Coloured in yellow }\end{array}$ & None \\
\hline Liver cutting area & $\begin{array}{l}\text { Intersection between the resection plane and the liver, dilated to } \\
\text { have a thickness of } 0.3 \mathrm{~cm} \text {. Coloured in red }\end{array}$ & Enable/disable \\
\hline Resection route & $\begin{array}{l}\text { Line drawn in the resection plane that shows the initial entry point } \\
\text { and direction that is expected in the resection procedure. Colou- } \\
\text { red in pink }\end{array}$ & None \\
\hline
\end{tabular}

\begin{tabular}{|c|c|c|}
\hline Component & Information visualisation and function & Interaction \\
\hline Surgical view & $\begin{array}{l}\text { Visualisation of the Resection Map with } \\
\text { the same orientation that the surgeon is } \\
\text { expected to find in the real procedure. } \\
\text { This view is parallel to the resection } \\
\text { plane }\end{array}$ & Rotation, zoom, reset to initial orientation \\
\hline Progress view & $\begin{array}{l}\text { Visualisation of the Resection Map from a } \\
\text { perpendicular direction to the resection } \\
\text { plane. It illustrates the resection route on } \\
\text { the } 2 D \text { grid }\end{array}$ & Rotation, zoom, reset to initial orientation \\
\hline Resection window & $\begin{array}{l}\text { Volume that defines the visualisation frus- } \\
\text { tum of surrounding structures to the } \\
\text { resection plane (except for tumours) }\end{array}$ & Increase/decrease window width \\
\hline Progress window & $\begin{array}{l}\text { Portion of the resection window at a point } \\
\text { of the Resection Route. It provides a clear } \\
\text { visualisation of the structures belonging } \\
\text { to it }\end{array}$ & Advance/back resection progress \\
\hline
\end{tabular}

progress with the sequential identification of landmarks. It also highlights the location of the Progress Window, for a better correspondence between Progress and Surgical views.

Therefore the main way of interaction with the Resection Map is to go back and forth along the resection progress, that is, to slide the Progress Window simultaneously in both views. Secondly, both views of the map can be rotated to offer perspective views that enhance the understanding of veins topology. Finally, some features can be enabled and disabled, like the 2D grid on the plane or the veins displayed in the Progress View.

\section{Integration in current surgical workflow}

The system can be used by surgeons in both open and laparoscopic surgery during the resection phase. As a first approach, an assistant is expected to manage the Resection Map interactivity following the voice commands of the surgeon, as it is currently done in some hospitals to navigate through CT slices. The Resection Map can be visualized in the 2D monitor screen already existing in the surgical theatre, and the computer running the software can be located elsewhere, for example by the terminal that controls the $\mathrm{CT}$ visualisation. It has to be noted that this is only our integration plan, since the Resection Map has not yet been tried in a real surgical scenario.

\section{Implementation and concept validation}

\section{First prototype construction}

A Resection Map can be generated from preoperative image studies (MR, CT). In the case of liver resection the most typical study is the contrast enhanced CT that enables the visualisation of vessels. The definition of the resection path and plane is done in a preoperative stage using a planning 
tool like those described in [3]. Once this plane is defined, the contents of the Resection Map can be generated: the resection plane, the main veins and the resection targets (tumours). The planned entry point and direction for the resection are also defined by the surgeon in this planning stage.

There are two main strategies for the visualisation of liver structures, segmentation and $3 \mathrm{D}$ reconstruction or volume rendering [15]. Considering the current technological state of art, an automatic segmentation is difficult to achieve for complex structures such as liver vascular system. Nevertheless, it leads to cleaner visualisation results and the possibility of quantitative analysis (diameter and connectivity of vessels, tumour size, etc.). Moreover, volume rendering visualisation often requires manual adjustment of transfer functions. These are the reasons that led us to take the segmentation and reconstruction option.

A contrast enhanced CT image study which contains several tumours is selected for a first prototype of the resection map. Images are acquired with a Somatom Emotion 16 (Siemens Medical Solutions, Malvern, PA, USA) with a voxel size of $(0.6 \times 0.6 \times 5) \mathrm{mm}$. Injected contrast was $100 \mathrm{ml}$ Omnipaque 300 (GE Healthcare, Chalfont St Giles, UK). Parenchyma and tumours are segmented using a coarseto-fine algorithm based on gradient vector flow snakes and patient specific statistics [16]. Vessels are segmented using a detection filter based on a multi scale medialness function, finding their centrelines and radii [17]. Three-dimensional surfaces of all structures are reconstructed with deformable simplex meshes [18].

Surgical planning of the resection is done with a simple straight plane, since the aim is to present a proof of concept rather than a complete system evaluation. This planning is then prepared for its intraoperative visualisation as shown in Figs. 3 and 4, which implement the specifications defined in former section. This visualisation tool is developed with Coin3D [19], an open source library based on Open Inventor. Three-dimensional reconstruction results are thus converted into Inventor files, which are then loaded and prepared for rendering using the classes of the library. The 3D visualisation windows are implemented with clipping planes. Perspective and orthographic camera models are respectively selected for the generation of the surgical and progress views.

\section{Preliminary acceptance results}

Preliminary testing of the prototype was conducted with surgeons $(n=5)$ in two national hospitals: Rikshospitalet (Oslo, Norway) and Hospital Clínico (Zaragoza, Spain). The methodology was personal/group guided interviews in which surgeons were presented with the Resection Map. This exploratory study revealed the following findings:
1. Increase in confidence: Surgeons found the idea of presenting the resection plan along with the key anatomical structures and risk areas very useful. Especially for the resident surgeons, this facility allows recalling the planned information.

2. Enhance of orientation: despite not characterising it as optimal, surgeons assessed the visualisation as a useful guidance tool.

3. The main application is in non anatomical resection. This is the most challenging situation for the surgeon, and where a Resection Map is most needed. The system should have the capability of displaying irregular resection planes.

4. Lack of positioning information: surgeons missed the possibility of knowing where they are in the Map, to automatically follow the progress of the resection.

\section{Discussion}

Surgical demand exists for computer aided surgical systems in both open and laparoscopic hepatectomies. Surgeons expect orientation and visualisation support during operations that allow for a more accurate and secure execution of the planned operation, especially in non anatomical resections. Conducted surgical requirements analysis supports surgeon centred technological development and minimizes the risk of unnecessary technology push in the OR.

Based on surgical requirements, an intuitive visualisation tool has been proposed in this paper as a pragmatic solution for intra-operative liver resection guidance. This Resection Map has some design issues that could be discussed. The most controversial one is probably the need of the relative positioning of surgical tools in the map, which surgeons have already mentioned is missing in our preliminary evaluation. Our proposal is to disregard it in a first step due to the extreme technical difficulty to acquire and register it due to the big deformations of the operating field.

The other main design aspect is to offer an intuitive and effective visualisation of liver structures. The solution proposed in this article is the result of several design iterations between engineers, experts in human factors and surgeons, but it probably still needs improvements. Perception of depth information in the surgical view of the Map is an issue, and current solution might also need the inclusion of more shading or other additional effects. It has also been discussed the inclusion of some colour code information about the proximity of veins to the resection plane. Finally, there are some design parameters to tune and adapt based on real cases, for example the minimum diameter of veins or the size of the $3 \mathrm{D}$ visualisation windows.

To our knowledge after reviewing the literature, this is one of the first efforts directed towards the effective intraoperative 
Fig. 3 Prototype of the Resection Map, displaying both the surgical (left) and the progress (right) views. The progress window is here a vertical box selecting the beginning of the resection, and it will slide following the pink resection route
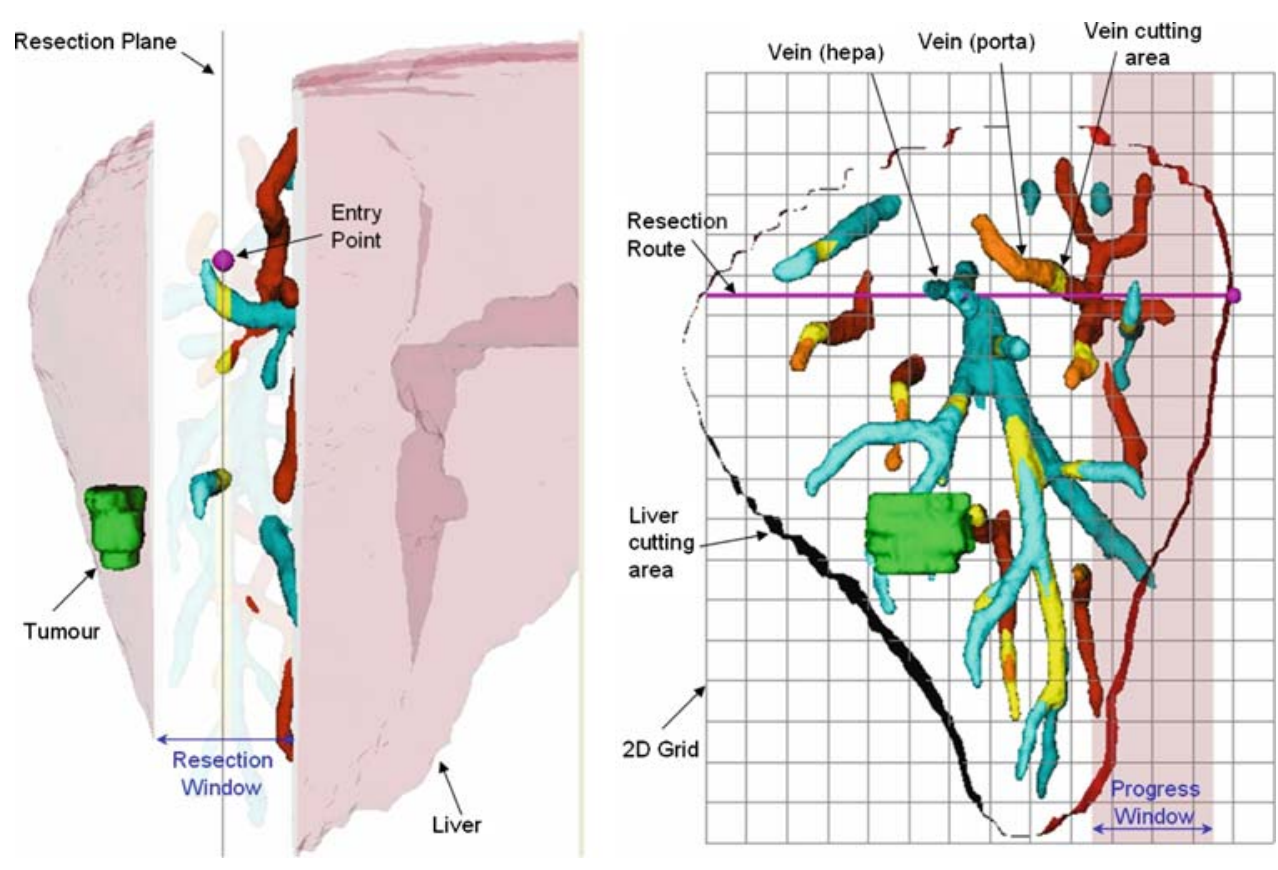

Fig. 4 Prototype of the Resection Map after some interactions with respect to the visualisation of Fig. 3. The progress window is moved $2.0 \mathrm{~cm}$ forward; the surgical view is rotated; veins are deactivated in the progress view, which clearly shows the relative localisation of the key landmarks (the vein cutting areas) that are found in the resection plane

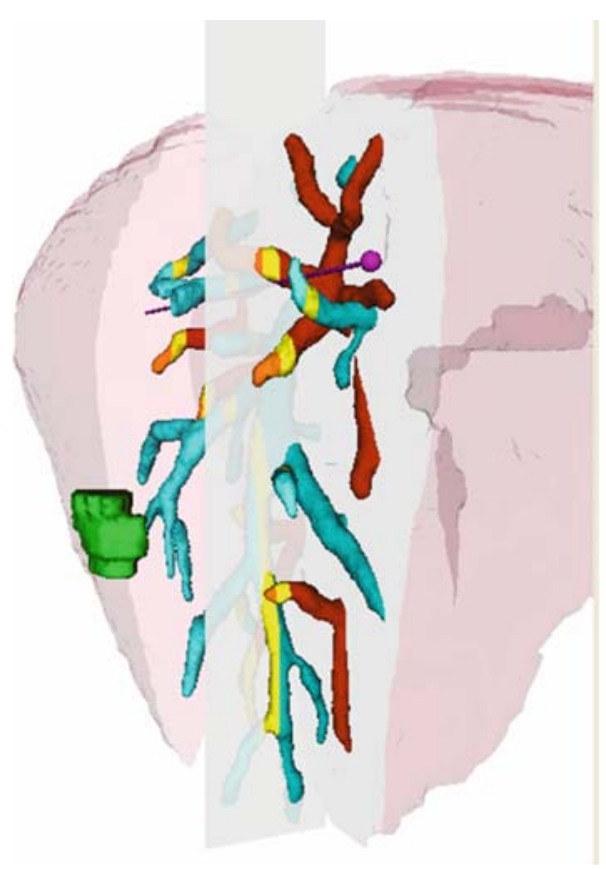

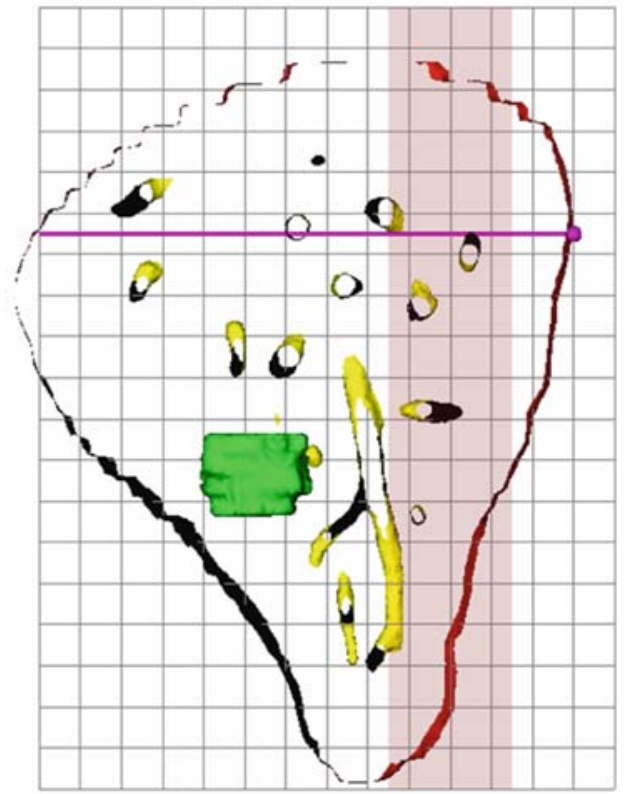

guidance of hepatectomies. Related works are focused in the preoperative stage. They provide clear representations of liver anatomy, and advanced interfaces to explore it and decide a resection plane. They can be also used in the OR [13], but they are not designed for the intraoperative requirements. We believe that the design of the Resection Map provides the necessary orientation information and confidence to the surgeon in order to perform a safer resection, progressing towards a solution to fill the existing gap between pre- and intra-operative visualisation.
The visualisation concept of the Resection Map, as a non-registered additional 3D window, is similar to the laparoscopic views of 3D CT angiography proposed for lymph node dissection [20]. In our opinion, a direct volume rendering of CT data used in this work does not provide a clean and intuitive visualisation. Segmentation and selection of key structures related to the surgical task are needed for an effective guidance.

There are some interesting extensions to this first proposal of the Resection Map focusing on the capability of updating 
the planning strategy. It could be simply the possibility of choosing among several resection scenarios planned before the surgery and based on intra-operative findings and judgments. The next step would be an interface for dynamically checking and updating the contents of the Resection Map based on new findings on the intraoperative ultrasound, in a similar fashion as described in [21]. Another direction could be the inclusion of more information, like the irrigated volume of each vessel (associated functionality loss) or the 3D structure of arteries and bile ducts.

Our next research steps are directed towards enhancing current prototype, especially for non anatomical resections which require non-straight resection planes. Clinical studies are planned to objectively assess the value of this guidance tool in open and laparoscopic hepatectomies, and to quantify the importance of displaying the relative positioning of surgical tools in the Map.

\section{Conclusion}

The Resection Map is proposed as a pragmatic solution to enhance liver resection accuracy and safety with an intuitive visualisation of its critical inner structures. Its design provides useful information for improving management of pre-operative knowledge during liver surgery, even if it does not yet include the positioning of surgical tools, and allows a seamless integration in the OR. Clinical studies are planned to objectively assess the value of this guidance tool in open and laparoscopic hepatectomies.

Acknowledgments We would like to thank the surgeons who took part of this project for their very helpful comments and guidance, especially to Dr B. Edwin (Rikshospitalet, Oslo, Norway) and to Dr V. Sojar (Ljubljana MC, Slovenia). This work is part of the ARIS*ER Marie Curie Research Training Network (MRTN-CT-2004-512400), which is funded by the Sixth Framework Programme of the European Commission. Our acknowledgement also to the ARIS*ER partners and colleagues who supported us in the Resection Map development work.

\section{References}

1. Lang H, Radtke A, Hindennach M et al (2005) Impact of virtual tumor resection and computer-assisted risk analysis on operation planning and intraoperative strategy in major hepatic resection. Arch Surg 140(7):629-638

2. Soler L, Delingette H, Malandain G et al (2001) Fully automatic anatomical, pathological, and functional segmentation from CT scans for hepatic surgery. Comput Aided Surg 6(3):131-142. doi:10.1002/igs.1016

3. Reitinger B, Bornik A, Beichel R, Schmalstieg D (2006) Liver surgery planning using virtual reality. IEEE Comput Graph Appl 26(6):36-47. doi:10.1109/MCG.2006.131

4. Meinzer H-P, Thorn M, Cardenas CE (2002) Computerized planning of liver surgery-an overview. Comput Graph 26(4):569-576. doi:10.1016/S0097-8493(02)00102-4
5. Ayache N (2003) Epidaure: a research project in medical image analysis, simulation, and robotics at INRIA. IEEE Trans Med Imaging 22(10):1185-1201. doi:10.1109/TMI.2003.812863

6. Jalote-Parmar A, Pattynama P, Goossens R, Freudenthal A, Samset E, de Ridder H (2006) Bridging the gap: a user centered design approach towards developing technological solutions. In: Casciaro S, Distante A (eds) Minimally invasive therapies and novel embedded technology systems. Lupiensis Biomedical Publications, pp 93-101

7. Jalote-Parmar A, Pattynama P, de Ridder H, Goossens R, Freudenthal A, Samset E (2007) Surgical workflow analysis: identifying user requirements for surgical information systems. In: Pikaar R, Ernst K, Settels (eds) Diversity in ergonomics. Elsevier, Oxford, pp 229-241

8. Beller S, nerbein M, Eulenstein S, Lange T, Schlag PM (2007) Feasibility of navigated resection of liver tumors using multiplanar visualization of intraoperative 3-dimensional ultrasound data. Ann Surg 246(2):288-294. doi:10.1097/01.sla.0000264233.48306.99

9. Feuerstein M, Mussack T, Heining SM, Navab N (2008) Intraoperative laparoscope augmentation for port placement and resection planning in minimally invasive liver resection. IEEE Trans Med Imaging 27(3):355-369. doi:10.1109/TMI.2007.907327

10. Scheuering M, Schenk A, Schneider A, Preim B, Greiner G (2003) Intraoperative augmented reality for minimally invasive liver interventions. In: Galloway RL Jr (ed) Proceeding of SPIE medical imaging, San Diego, vol 5029, pp 407-417. doi:10.1117/12.480212

11. Jalote-Parmar A, Pattynama P, Van der Plas A, Heer P (2008) Towards intuitive surgical interfaces: strategies from video games. In: Casciaro S, Distante A (eds) New technology frontiers in minimally invasive therapies. Lupiensis Biomedical Publications, pp $101-111$

12. Khan AZ, Mudan SS (2007) Liver regeneration: mechanisms, mysteries and more. ANZ J Surg 77(1-2):9-14. doi:10.1111/j. 1445-2197.2006.03981.x

13. Grenacher L, Thorn M, Knaebel HP et al (2005) The role of 3 -D imaging and computer-based postprocessing for surgery of the liver and pancreas. Rofo 177(9):1219-1226

14. Kalkofen D, Mendez E, Schmalstieg D (2007) Interactive focus and context visualization for augmented reality. In: Proceeding of ISMAR, international symposium on mixed and augmented reality, Nara, pp 191-200. doi:10.1109/ISMAR.2006.297816

15. Freiman M, Joskowicz L, Lischinski D, Sosna J (2007) A featurebased transfer function for liver visualization. Int J CARS 2(Supp 1):125-126

16. Casciaro S, Massoptier L, Samset E, Casciaro E, Distante A (2006) A method for fast and automatic segmentation of soft organs from CT and MR images. Int J CARS 1(Supp 1):470-471

17. Pock T, Beichel R, Bischof H (2005) A novel robust tube detection filter for 3D centerline extraction. In: Lecture notes in computer science, vol 3540, pp 481-490. doi:10.1007/11499145_49

18. Bornik A, Reitinger B, Beichel R (2005) Simplex-mesh based surface reconstruction and representation of tubular structures. Bildverarbeitung für die Medizin, pp 143-147. Springer, Heidelberg. doi:10.1007/3-540-26431-0_30

19. Coin 3D (2008). http://www.coin3d.org

20. Takiguchi S, Sekimoto M, Fujiwara Y et al (2004) Laparoscopic lymph node dissection for gastric cancer with intraoperative navigation using three-dimensional angio computed tomography images reconstructed as laparoscopic view. Surg Endosc 18(1):106-110. doi:10.1007/s00464-003-8116-y

21. Hansen C, Schlichting S, Zidowitz S et al (2008) Intraoperative adaptation and visualization of preoperative risk analyses for oncologic liver surgery. In: Miga MI, Cleary KR (eds) Proceedings of medical imaging 2008: visualization, image-guided procedures, and modeling, vol 6918. SPIE, San Diego (in press). doi:10.1117/ 12.770243 